\title{
EVALUATION OF FOUR PHENOTYPIC TESTS FOR THE DETECTION OF OXA-48-TYPE CARBAPENEMASE IN ENTEROBACTERIACEAE
}

\author{
Daniela Talapan ${ }^{1,2}$, Olga Mihaela Dorobat ${ }^{1}$, Coralia Bleotu $^{3}$, Ioana Aldea ${ }^{3}$, \\ Laura Necula ${ }^{3}$, Dragos Florea ${ }^{1,2}$, Gabriel Adrian Popescu ${ }^{1,2}$, \\ Adrian Streinu-Cercel ${ }^{1,2}$, Alexandru Rafila ${ }^{1,2}$ \\ 1 "Prof. Dr. Matei Bals" National Institute of Infectious Diseases, Bucharest \\ 2 "Carol Davila" University of Medicine and Pharmacy, Bucharest \\ ${ }^{3}$ Stefan S. Nicolau Institute of Virology, Romanian Academy, Bucharest
}

\begin{abstract}
Carbapenemase-producing Enterobacteriaceae have the potential to rapid dissemination in healthcare settings, becoming a major infection control and public health concern. PCR remains the reference method for identifying these strains, but is still expensive. We evaluated the performance of four phenotypic tests for identifying OXA-48-producing (Oxacillinase-48) Enterobacteriaceae. These were modified Hodge test (MHT), a combination disk test with meropenem alone or in combination with various inhibitors and temocillin, an enzymatic test and an immunochromatographic test. The tests were performed and interpreted according to guidelines or manufacturer's recommendations. The most frequent microorganism included in the study was Klebsiella pneumoniae. All the tests had $100 \%$ specificity, except MHT (87,5\%). Sensitivity was $91,25 \%$ for $\mathrm{MHT}, 90 \%$ for the combination disk test, $95 \%$ for the enzymatic test and $82,50 \%$ for the immunochromatographic test.
\end{abstract}

Keywords: Enterobacteriaceae, OXA-48 carbapenemase, phenotypic tests

\section{INTRODUCTION}

The resistance to carbapenems may be achieved by impermeability plus hiperproduction of AmpC or by producing carbapenemase, an enzyme that hydrolases the carbapenem. There are 3 classes of carbapenemase of great clinical importance: A, B and D according to the Ambler molecular classification system. The D class consists of OXA-48-type beta-lactamases (oxacillinase) and they are plasmid-encoded, which increases the risk of spread amongst microorganisms and in healthcare facilities (1).

Since the first isolation of OXA-48-carbapenemase in Turkey in 2001, in a Klebsiella pneumoniae isolate (2), this type of carbapenemase was spread throughout Europe (3-7), Middle East (8-10) and North America (11).

The aim of our study was to evaluate four phenotypic tests for the detection of OXA-48 carbapenemase in Enterobacteriaceae. PCR remains the reference method for identifying carbapenemase-producing bacterial strains, but it is still expensive and requires well-trained personnel.

\section{MATERIAL AND METHODS}

Between October 2013 and July 2015, there were isolated Enterobacteriaceae strains with decreased susceptibility to one or more carbapenems according to EUCAST (European Committee on Antimicrobial Susceptibility Testing) 
guidelines from patients and carriers admitted to the National Institute for Infectious Diseases "Prof. Dr. Matei Bals". Identification and antibiotic susceptibility testing were performed using Gram negative Combo 56 panels on MicroScan Walk Away system (Siemens, USA). In our study, there were selected 112 non-duplicate Enterobacteriaceae strains, well characterized by molecular tests (PCR): 80 strains were positive for bla $_{\text {OXA-48 }}$ gene and 32 were non-carbapenemase producers.

The studied phenotypic tests were MHT (recommended by CLSI - Clinical and Laboratory Standards Institute), KPC (Klebsiella pneumoniae carbapenemase), MBL (Metallo-beta-lactamase) and OXA-48 Confirm kit (Rosco Diagnostica, Denmark), Rapidec ${ }^{\circledR}$ Carba NP (bioMerieux, France) (both recommended by EUCAST) and OXA-48 $\mathrm{K}$-SeT (Coris BioConcept, Belgium).

\section{MHT}

Modified Hodge test was performed and interpreted according to CLSI guideline using Klebsiella pneumoniae ATCC BAA 1705 as positive control (12). A suspension of $1 / 10$ from 0,5 McFarland E. coli ATCC 25922 was inoculated on Mueller Hinton E agar (MHE, bioMerieux, France). There was used a $10 \mu \mathrm{g}$ of ertapenem disk and bacterial strains were inoculated in straight line from antibiotic disk to the edge of the plate. The apearance of the cover leaf after incubation at $35^{\circ} \mathrm{C}$ for $18-20$ hours indicates the of carbapenemase production.

\section{KPC, MBL and OXA-48 Confirm kit}

This kit contains tablets of meropenem (10 $\mu \mathrm{g}$ ) alone or plus inhibitors for various types of beta-lactamase: meropenem $(10 \mu \mathrm{g})$ plus Phenylboronic Acid (KPC and AmpC), meropenem $(10 \mu \mathrm{g})$ plus Dipicolinic Acid (MBL), meropenem $(10 \mu \mathrm{g})$ plus Cloxacillin (AmpC) and Temocillin $(30 \mu \mathrm{g})$. The test was performed and interpreted according to manufacturers' recommendations. The 0,5 McFarland of bacterial strain to be tested was inoculated on MHE agar and the tablets were placed at equal distance one from the other. The plates were incubated at $35^{\circ} \mathrm{C}$ for 18 20 hours and read by measuring the diameter of the inhibition zone (in $\mathrm{mm}$ ) around each tablet. No inhibition zone around temocillin indicates the of OXA-48-type carbapenemase-producing.

\section{Rapidec ${ }^{\circ}$ Carba NP}

This is an enzymatic approach to identify the carbapenemase-producers. The kit contains imipenem (carbapenemase substrate), phenol red (as $\mathrm{pH}$ indicator) and zinc (for the detection of MBL-producing strains). Fresh bacterial strains from COLS (Columbia agar plus sheep blood, Oxoid, UK) or MHE were used to perform the test as recommended by the manufacturer. The results were red after 30 minutes and/or another 1 hour and 30 minutes of incubation at $37^{\circ} \mathrm{C}$. Changing the colour from red (negative control) to yellow, orange-yellow or orange-red in the test was an indicator of a carbapenemaseproducing process.

\section{OXA-48 K-SeT}

This test was released on the market in 2015 and it is based on a membrane technology with colloidal gold nanoparticles conjugated with a monoclonal antibody directed against one epitope of the OXA-48 carbapenemase and another monoclonal antibody directed against another epitope of OXA-48 carbapenemase attached on a nitrocellulose membrane.

It can detect OXA-48-like carbapenemases from a single colony of Enterobacteriaceae isolated on agar plate. The test consist of lysing buffer and cassetts. After inoculating the bacterial colony in the buffer, add 3 full drops of mixture into the sample well of a cassette and read after 15 minutes.

The performance of the phenotypic tests for the detection of carbapenemase-producers was determined using the genotypic method as gold standard. Sensitivity was calculated from the 
number of true-positive isolates and specificity from the number of true-negative isolates.

\section{RESULTS}

The bacterial strains included in this study were almost equally isolated from women $(n=53)$ and men $(n=59)$. Most of the strains $(78,57 \%)$ were isolated from patients aged 51 years old.

The isolates were from patients and carriers (Table 1). Among the strains isolated from patients, $75 \%$ were carbapenemase-producers, while from carriers were $55 \%$. All the strains isolated from the lower respiratory tract infections were bla $a_{\text {OXA-48 }}$ positive and 5 out of 6 from blood. A percent of 74,6 of the strains isolated from urine were producing OXA-48 carbapenemase.

TABLE 1. The isolates

\begin{tabular}{|l|l|l|l|}
\hline $\begin{array}{l}\text { Clinical } \\
\text { specimens }\end{array}$ & Carba positive & $\begin{array}{l}\text { Carba } \\
\text { negative }\end{array}$ & Total \\
\hline Urine & 47 & 16 & 63 \\
\hline Sputum & 9 & 0 & 9 \\
\hline Pleural fluid & 1 & 0 & 1 \\
\hline Wound & 7 & 5 & 12 \\
\hline Blood & 5 & 1 & 6 \\
\hline $\begin{array}{l}\text { Sonication } \\
\text { fluid }\end{array}$ & 0 & 1 & 1 \\
\hline Rectal swab & 11 & 9 & 20 \\
\hline
\end{tabular}

The isolated bacterial strains were as follows: Klebsiella pneumoniae (n=95), E. coli $(\mathrm{n}=6), E n$ terobacter cloacae $(\mathrm{n}=5)$, Enterobacter aerogenes $(\mathrm{n}=1),(\mathrm{n}=4)$ and Morganella morgannii $(\mathrm{n}=1)$. Among Klebsiella pneumoniae strains, 74,73\% were OXA-48-positive and no Enterobacter cloacae and Morganella morgannii were found. Five out of 6 E. coli and 3 out of 4 Serratia marcescens were carbapenemase-producers. The Enterobacter aerogenes strain was also positive for OXA-48.

After performing MHT, 7 out of 80 strains were false negative and 4 out of 32 were false positive carbapenemase-producers. The combination disk test (Rosco Diagnostica) missed to identify 8 out of 80 positive OXA-48, but identified all non-carbapenemase-producers. The enzymatic test had 4 false negative results and the immunochromatographic test had 14 false negative results, both tests correctly identified non-carbapenemase-producers.

The sensitivity and specificity of each phenotypic test is summarized in Table 2 .

TABLE 2. The sensitivity and specificity of phenotypic tests

\begin{tabular}{|l|l|l|l|l|l|}
\hline $\begin{array}{l}\text { Test } \\
\text { Result }\end{array}$ & $\begin{array}{l}\text { PCR } \\
\text { blaOXA-48 }\end{array}$ & MHT & $\begin{array}{l}\text { KPC, MBL } \\
\text { and } \\
\text { OXA-48 }\end{array}$ & $\begin{array}{l}\text { Rapidec } \\
\text { CarbaNP }\end{array}$ & $\begin{array}{l}\text { OXA-48 } \\
\text { K-SeT }\end{array}$ \\
\hline Positive (no.) & 80 & 73 & 72 & 76 & 66 \\
\hline Negative (no.) & 32 & 28 & 32 & 32 & 32 \\
\hline Sensitivity (\%) & 91.25 & 90 & 95 & 82.5 \\
\hline Specificity (\%) & & 87.5 & 100 & 100 & 100 \\
\hline
\end{tabular}

\section{DISCUSSIONS}

As from literature, carbapenem non-susceptible strains were isolated from old patients who most probably had antimicrobial treatment in the past. More than three quarters were more than 50 years old.

Most of the strains with decreased susceptibility to carbapenems and carbapenemaseproducers were Klebsiella pneumoniae in our study, in accordance with data from the literature $(13,14)$.

Among the phenotypic tests studied, MHT is not recommended by EUCAST guidelines, due to low sensitivity and specificity. We found the lowest specificity amongst the phenotypic tests studied and a good sensitivity (91.25\%) in detecting OXA-48-producers, close to the results from literature: $100 \%$ sensitivity by Girlich D et al (15), 94\% by Bartolini A et al (14) and 93\% by Doyle D et al (16).

The KPC, MBL and OXA-48 Confirm kit had $100 \%$ specificity and $90 \%$ sensitivity in detecting OXA-48 producers in our study. The specificity of the test was the same as in the study of van Dijk et al (100\%), but with less sensitivity (90\% versus 100\%) (17).

Rapidec CarbaNP had in our study 100\% specificity, like in Dortet L. et al study, but less 
sensitivity (95\% versus 99\%) (18). In a study by Kabir MH et al, the sensitivity was $97,8 \%$ and specificity $98,56 \%$ (19).

The newest phenotypic test introduced on the market for the detection of OXA-48-type carbapenemase in Enterobacteriaceae is OXA-48 $\mathrm{K}$ SeT. Despite the results found in the literature of being $100 \%$ sensitive and specific $(20,21)$, our results showed less sensitivity $(82,5 \%)$, maybe because of the detection limit $(0,125 \mathrm{ng} / \mathrm{ml})$, the OXA-48-producing strains being known to be weak carbapenemase-producers.

\section{CONCLUSIONS}

Among the phenotypic tests with 100\% specificity, Rapidec CarbaNP had the highest sensitivity for the detection of OXA-48 carbapenemase producers. It is also a test which gives the results in 2-4 hours, being very useful in the rapid identification of such strains and triggering immediately the infection control policies in order to minimize the spread in healthcare facilities.

KPC, MBL and OXA-48 Confirm kit has a good sensitivity for OXA-48 producers, but the result is available after 18-20 hours.

Despite its low specificity, MHT remains useful due to the technical availability and good sensitivity for the detection of OXA-48 carbapenemase producing strains.

OXA-48 $K$-SeT is a rapid and highly specific test, but showed low sensitivity on our strains.

Acknowledgements: This paper was supported by the Sectorial Operational Program for Human Resources Development (SOP HRD), financed from the European Social Fund and by the Romanian Government under the contract number POSDRU/159/1.5/S/137390/.

\section{REFERENCES}

1. Queenan A.M., Bush K. Carbapenemases: the Versatile ß-Lactamases. Clin Microbiol Rev 2007; 20(3): 440-458.

2. Poirel L., Heritier C., Tolun V., Nordmann P. Emergence of oxacillinase-mediated resistance to imipenem in Klebsiella pneumoniae. Antimicrob Agents Chemother 2004; 48: 15-22

3. Pitart C., Sole M., Roca I. et al. First outbreak of a plasmid-mediated carbapenem-hydrolyzing OXA-48 beta-lactamase in Klebsiella pneumoniae in Spain. Antimicrob Agents Chemother 2011; 55: 4398-401

4. Levast M., Poirel L., Carrer A. et al. Transfer of OXA-48-positive carbapenem-resistant Klebsiella pneumoniae from Turkey to France. J Antimicrob Chemother 2011; 66: 944-5

5. Glupczynsky Y., Huang T.D., Bouchahrouf W. et al. Rapid emergence and spread of OXA-48-producing carbapenem-resistant Enterobacteriaceae isolates in Belgian hospitals. Int J Antimicrob Agents 2012; 39: 168-172

6. Canton R., Akova M., Carmeli Y. et al. Rapid evolution and spread of carbapenemases among Enterobacteriaceae in Europe. Clin Microbiol Infect 2012; 18: 413-31

7. Voulgari E., Zarkotou O., Ranellou K. et al. Outbreak of OXA-48 carbapenemase-producing Klebsiella pneumoniae in Greece involving an ST11 clone. J Antimicrob Chemother 2013; 68: 84-8

8. Ktari S., Mnif B., Louati F. et al. Spread of Klebsiella penumoniae isolates producing OXA-48 $\beta$-lactamase in a Tunisian university hospital. J Antimicrob Chemother 2011; 66: 1644-1646

9. Shibl A., Al-Agamy M., Memish Z. et al, The emergence of OXA-48- and NDM-1-positive Klebsiella pneumoniae in Riyadh, Saudi Arabia. Int J of Infect Dis 2013; 17: 1130-1133

10. Beyrouthy R., Robin F., Dabboussi F. et al. Carbapenemase and virulence factors of Enterobacteriaceae in North Lebanon between 2008 and 2012; evolution via endemic spread of OXA-48. J Antimicrob Chemother, 2014;

11. Mathers A.J., Hazen K.C., Caroll J. et al. First clinical cases of OXA-48-producing carbapenem-resistant Klebsiella pneumoniae in United States: the "menace" arrives in the new world. J Clin Microbiol 2013; $51: 680-683$

12. Clinical and Laboratory Standards Institute. Performance standards for antimicrobial susceptibility testing: $25^{\text {th }}$ informational supplement. CLSI M100-S25. 2015, Wayne, PA

13. Song W., Hong S.G., Yong D. et al. Combined use of the modified Hodge test and carbapenemase inhibition test for detection of carbapenemase-producing Enterobacteriaceae and metallo- $\beta$ lactamase producing Pseudomonas spp. Ann Lab Med, 2015; 35: 212-219

14. Bartolini A, Frasson I, Cavallaro A et al, Comparison of phenotypic methods for the detection of carbapenem non-susceptible Enterobacteriaceae. Gut Pathogens 2014; 6:13

15. Girlich D, Poirel L and Nordmann $P$, Value of the Modified Hodge Test for detection of emerging carbapenemases in Enterobacteriaceae. J Clin Microbiol 2012; 50(2): 477-479 
16. Doyle D., Peirano G., Lascols C. et al. Laboratory detection of Enterobacteriaceae that produce carbapenemases. J Clin Microbiol 2012; 50(12): 3877-80

17. Van Dijk K., Voets G.M., Scharringa J. et al. A disk diffusion assay for detection of class A, B and OXA-48 carbapenemases in Enterobacteriaceae using phenyl boronic acid, dipicolinic acid and temocillin. Clin Microbiol Infect 2014; 20: 345-349

18. Dortet L., Agathine A., Naas T. et al. Evaluation of the Rapidec ${ }^{\circledR}$ Carba NP, the Rapid Carb Screen ${ }^{\circledR}$ and the Carba NP test for biochemical detection of carbapenemase-producing Enterobacteriaceae. J Antimicrob Chemother 2015; 70(11): 3014-22

19. Kabir M.H., Meunier D., Hopkins K.L. et al. A two-centre evaluation of Rapidec ${ }^{\circledR}$ Carba NP for carbapenemase detection in
Enterobacteriaceae, Pseudomonas aeruginosa and Acinetobacter spp. J Antimicrob Chemother 2016;71(5): 1213-6

20. Wareham D.W., Shah R., Betts J.W. et al. Evaluation of an Immunochromatographic Lateral Flow Assay (OXA-48 K-SeT) for rapid detection of OXA-48-like carbapenemases in Enterobacteriaceae. J Clin Microbiol 2016; 54(2): 471-473

21. Dortet L., Jousset A., Sainte-Rose V. et al. Prospective evaluation of the OXA-48 K-SeT assay, an immunochromatographic test for the rapid detection of OXA-48-type carbapenemases. J Antimicrob Chemother 2016; 71(7): 1834-40 